\title{
A propos du Dr Jean de Carro
}

\author{
Par le Dr Jean Olivier, Genève
}

Notre petit travail n'a pas d'autre prétention que d'apporter un complément à l'intéressante étude que le Professeur Sigerist a publiée dans le numéro 12 des «Supplements to the Bulletin of History of Medecine», en 1950, en tête de vingt deux lettres de DE CARRo à son ami et compatriote, le Dr Marcet, établi à Londres.

Rappelons brièvement que le Dr JeAn dE CArro, de très ancienne souche genevoise, après des études à Edimbourg, revint dans sa ville natale au pire moment de la Terreur, ce qui le décida à gagner Vienne, où il pratiqua jusqu'en 1826; il se rendit alors à Carlsbad, qui, grâce à ses adroites réclames, acquit la renommée qu'on lui connaît. Il s'intéressa à des questions fort diverses, mais il faut garder le souvenir des grands services qu'il rendit à l'humanité en répandant la vaccination dans l'Est de l'Europe et jusqu'au plus lointain Orient.

Ce qu'il raconte de lui-même, et non sans forfanterie, tant dans ses Mémoires que dans sa correspondance, donne envie d'être renseigné par des personnes qu'il a rencontrées et c'est ce que nous avons cherché à tirer de quelques documents inédits.

Le Professeur Sigerist, dans son étude, a très bien défini ce qu'on peut conclure du caractère de DE CARRo d'après ses actes: "He was not only a good doctor, but also a great promoter and salesman. This is evidenced by his entire career. He had an extremely alert mind that was always on the lookout for some discovery that he could adopt, propagate and exploit. He never made a discovery himself, but he usually showed good judgement in taking up other people's findings."

De Carro avait la plume abondante et facile; on s'en rend compte, par exemple, dans les cinquante lettres qui parurent sous sa signature dans la Bibliothèque Britannique et la Bibliothèque Universelle de Genève; plus de la moitié sont relatives à la vaccination, mais les autres ont trait aux sujets les plus divers: thermolampes, ver de Guinée et scorpions, chèvres de Russie, décomposition des alcalis, aérolithes, culture du riz sec, pharmacie portative du Thibet et tant d'autres pour finir par les fumigations sulfureuses et le traitement du goitre par l'iode. Il va sans dire qu'en toute naïveté, dE CARRo ne s'y épargne pas les compliments! Il s'en faisait aussi volontiers décerner par des confrères de passage qu'il savait adroitement 
endoctriner. Nous pensons, par exemple, au Dr André-Louis Gosse (1791-1873) qui lui ressemblait sous certains rapports et qui rédigea pour la Bibliothèque Universelle un article fort élogieux sur les fumigations sulfureuses installées dans la maison de DE CARro à Vienne. Gosse avait une tendance à s'emballer, comme DE CARRo, mais il se différenciait de lui en ce sens que, d'abord, il avait lui-même des idées originales, puis que jamais il ne chercha à en tirer parti; il y dépensait, au contraire, et sans compter, son temps et sa bourse; on lui doit une série de travaux fort sérieux et il fit partie longtemps des Conseils de Genève. Sa voie était donc plus parallèle que semblable à celle de DE CARRo et ce qu'il y avait de divergent dans ces deux caractères aide à confirmer l'impression que l'on a de celui du médecin viennois.

Dans nos recherches de documents inédits, nous avons trouvé d'abord, au service des manuscrits de la Bibliothèque Universitaire de Genève, une série de quinze très longues lettres de DE CARro à l'historien genevois James Augustin Galiffe; cette correspondance date de 1814 à 1820 et est presque exclusivement relative à des questions de généalogie; on y voit avec quelle passion, notre confrère cherche à faire établir la noblesse et l'ancienneté très réelles de sa famille dans le but d'obtenir en Autriche un titre de chevalier: «même le titre de baron, écrit-il, mais le premier ne vaut guère plus que celui de simple gentilhomme et celui de baron ne convient pas trop à ma fortune ... ce qui joint aux mérites du premier introducteur de la vaccination sur le continent, et, par conséquent en Autriche; du riz sec (oryza mutica DE CARRo) et quelques services essentiels rendus à l'agriculture autrichienne par l'introduction des superbes merinos de Lancy; et à l'histoire d'Autriche par la traduction du Plutarque Autrichien et la mention des présents de la Compagnie des Indes, du gouverneur de Bombay, des Hospodats de Moldavie et de Valachie pour la vaccination orientale, et de la tabatière de MARIE-Louise, alors impératrice des Français, pour le Plutarque autrichien dont elle avait agréé la dédicace la veille de son départ de France, ne laissent pas que de faire un diplome dont mes enfants me sauront gré un jour, en nous procurant la qualité de gentilshommes autrichiens, confirmant à jamais par un seul acte l'ancien état de notre famille à Genève.»

Le déploiement de cette naĩve fatuité n'empêche pas DE CARro de déclarer dans une lettre suivante: «Vous savez, mon cher ami et compatriote, que la fumée des vanités de ce monde n'est pas celle qui m'occupe le plus...» En effet, c'est le moment où il a d'autres préoccupations plus matérielles: 
«... depuis le 24 juillet 1817 , je débite celle du soufre, avec profusion, au grand bénéfice de mon prochain et de moi-même ... Jusqu'à présent du moins mon établissement prospère, c'est à dire que les résultats médicaux et pécuniaires en sont très satisfaisants et que je trouve fort doux de gagner mon pain à la sueur du visage d'autrui ....»

Ces extraits de lettres assez amusantes ne nous donnent toujours que des impressions personnelles. Voyons si nous trouvons quelquechose de plus impartial chez des tiers!

Tout d'abord que pensaient de lui ses confrères genevois? Calmes, pondérés, objectifs, ils devaient se méfier un peu de ce compatriote désaxé, trop exubérant et superficiel; on s'en rend compte en lisant le procès-verbal de la séance du 20 août 1822 de la Société medico-chirurgicale: «M. DE CANdolle, président, lit une lettre de M. DE CARRo, médecin à Vienne, lequel, persuadé que les varioles récidives de Genève tiennent à la dégénération du vaccin primitif, propose d'envoyer de Vienne du vaccin; il se fonde sur ce que, dès l'an 1799 jusqu'à ce jour, il n'y a pas eu, dans les Etats autrichiens, un seul cas de récidive, ce qui, dans son opinion, prouve la supériorité de sa pureté. La Société pense que l'inoculation faite à Genève avec le vaccin de Vienne ne peut avoir aucun inconvénient, mais elle juge que M. DE CARRo s'abuse sur la cause des succès de la vaccine en Autriche ... M. MaunoIr aîné observe relativement à la proposition de M. DE CARRo d'envoyer de son vaccin infaillible en remplacement de celui de Genève qui a, dit-il, perdu de son efficacité, que le Dr Schauff, de Vienne, homme très judicieux et bien informé, lui a rapporté qu'un grand nombre de cas de petite vérole récidive avaient eu lieu dans cette dernière ville .... Rappelons que les médecins genevois avaient été, pour une bonne part, les pionniers de la vaccination sur le continent!

Nous voici maintenant, en 1814-1815 au Congrès de Vienne, source de beaux profits pour la vanité et pour la bourse du Dr DE CARRo, qui a, sur ses confrères, l'avantage de parler plusieurs langues. Il a écrit à MARCET: «Je suis, comme de tout temps, le médecin de préférence des anglais, ce qui n'empêche pas qu'une lettre de recommandation de ta part ne fasse toujours un bon effet quand l'occasion se présente à toi d'en donner aux anglais partant de Londres pour Vienne ... P Puis il annonce qu'il se prépare à accueillir les délégués genevois; il sera, en effet, un peu leur homme de confiance et le gouvernement de la République leur adressera la correspondance officielle sous son couvert. De Carro parle de Pictet de Roche- 
MONT, chef de la délégation, comme d'un de ses intimes amis, mais on verra que ce dernier ne partageait peut-être pas cette manière de voir!

Les délégués sont accompagnés de M. et Mme Jean Gabriel Eynard, neveux de Pictex, qui tinrent l'un et l'autre un journal de leur séjour à Vienne. Celui du mari a été publié en 1914 par M. Chapuisat : «Nous avons tourné et retourné dans tous les sens, écrit-il à l'arrivée, et enfin nous sommes parvenus à trouver la demeure du docteur DE CARRo qui nous a menés à un logement, composé de trois pièces assez propres qu'il a obtenues avec beaucoup de peine pour 600 florins par mois . . .» et le lendemain: «A deux heures nous avons été dîner chez M. DE CARRo qui nous a reçus avec sa cordialité ordinaire .... Ces entrevues se répéteront mais sans nous donner assez de détails pour avoir un portrait de DE CARRo.

Quant au journal de Madame Eynard, il est inédit et c'est grâce à l'obligeance de M. JACQues LE Fort que nous avons pu en prendre connaissance. Comme il n'y est pas question de diplomatie, nous espérions y trouver des impressions sur l'obligeant docteur, mais Mme EyNARD voit tant de monde plus intéressant qu'elle ne songe pas à faire autre chose que de citer sa présence au réunions de l'après-midi ou du soir. En effet, elle a un succès extraordinaire, danse avec l'empereur de Russie, avec des rois et des archiducs, avec Eugìne de Beauharnais, dîne chez Talleyrand et Metternich, est reçue à la cour et nous décrit de façon charmante cette vie mondaine.

C'est dans les lettres de Pictex de Rochemont à sa fille Amélie que nous allons enfin trouver un tableau de notre DE CARRo; une partie seulement de cette correspondance a paru dans l'ouvrage de J. B. GalIFFE, D'un siècle à l'autre, mais ce que nous rapportons ici se trouve dans les pages inédites que nous devons à l'amabilité de M. Marc Cramer : «. . . Vous voulez que je vous parle du brave DE CARro. Il ressemble à son frère en sinistre. Ce mot est le caractère de sa physionomie. Il sourit peu et ne rit guère. Il tourne les yeux portentously et allonge son visage à l'infini quand il veut donner du poids et de la gravité à ce qu'il dit. Il écoute beaucoup, c'est à dire trop et se répète comme il arrive à ceux qui ont cette maladie. Il n'a pas de trait et il manque de grâce dans l'esprit plus que je n'aurais cru. En tout, il tire sur le pesant et nos merveilleux (les EYNARD) trouvent que quatre fois par semaine au thé, c'est trop. La petite drôle de cousine (Mme EynarD) ne manque pas de me jeter un regard ou de me pousser du pied quand elle voit venir une histoire, surtout si elle a été faite. D'ailleurs, c'est le plus obligeant des hommes. Il est pour nous tout ce qu'on peut être 
sans être amusant, ce qui me donne du remords de ne pas le trouver tel. Il est fort estimé ici moralement et fort connu. Sa belle-mère qui est restée avec lui (grand avantage pour tous deux) en parle comme du plus excellent des hommes....

Ainsi de Carro était ennuyeux! Il se prenait sans doute trop au sérieux, s'appesantissait dans ses récits, se donnait de l'importance. Mais, ses emballements et ses fanfaronnades mis à part, c'était un homme dévoué et sincère; fat, snob, dirions-nous aujourd'hui; intéressé, il est vrai, mais honnête; adroit, pas bête, mais pas très intelligent non plus. Sa plus grande chance fut de rencontrer sur sa route, au début de sa carrière, la vaccination et de s'y consacrer avec un zèle et une adresse qui lui valent la reconnaissance très méritée de la postérité.

\section{Francesco Camuzios Consilium über das Steinleiden}

\section{Von Charles Salzmann, Zürich}

In der Manuskriptensammlung der Zentralbibliothek Zürich findet sich in Mscr. Sim. 204 $\mathbf{c}^{1}$, einem medizinischen Sammelband aus dem Besitze Conrad Gessners, ein Consilium des Luganeser Arztes Francesco Camuzıos, zusammen mit anderen ähnlichen Consilia gegen das Steinleiden, Abschriften von unbekannter Hand. Diese lateinische, undatierte Abschrift, als ein Consilium abgefaßt, dürfte einer früheren Zeit angehören, vor 1550. Der Sammler dieser Schriften ist unbekannt, noch viel weniger weiß man, wer sie C. Gessner schenkte. Die erwähnte Sammlung von Ratschlägen gegen das Steinleiden enthält folgende Beiträge : zwei vom Graf von SoLms, eine von Casarate «uff Italia», eine von Hieronymus Graf von Rapperswil, jene des Frangesco Camuzio, und eine von Vadian ${ }^{2}$. Aus dieser $\mathrm{Zu}$ sammenstellung kann man annehmen, daß der Sammler im ostschweizerischen oder süddeutschen Raume zu suchen wäre. Im dritten Bande des Thesaurus Medicinae Practicae (Mscr. Sim. 204c) sammelte G. Gessner neben eigenen Notizen, Krankengeschichten, Konsilien, Rezeptarien, Be-

${ }^{1}$ Mscr. Sim. 204 c Z. B.Zch.

2 B. Mrut, Ein Konsilium Vadians, Schw. Med. Wschr. 62.Jg. (1932), Nr. 11. Herrn Prof. Dr. B. Mrrt bin ich für den Hinweis auf den Camuzio-Text und manche Angaben zu Dank verpflichtet. 